\title{
Adipose tissue-derived stem cells attenuate acute lung injury through eNOS and eNOS-derived NO
}

\author{
PENG GAO $^{1}$, XU YANG $^{2}$, LUCKSHMANRAJ MUNGUR $^{2}$, SYLVANUS KAMPO $^{2}$ and QINGPING WEN $^{2}$ \\ ${ }^{1}$ Department of Anesthesiology, Dalian Medical University, Dalian, Liaoning 116044; ${ }^{2}$ Department of Anesthesiology, \\ The First Affiliated Hospital of Dalian Medical University, Dalian, Liaoning 116011, P.R. China
}

Received December 23, 2012; Accepted February 14, 2013

DOI: $10.3892 / \mathrm{ijmm} .2013 .1328$

\begin{abstract}
Acute lung injury (ALI) is among the most common causes of mortality in intensive care units. Recent in vivo and in vitro studies have suggested that mesenchymal stem cells (MSCs) attenuate pulmonary edema and inflammatory factors, but the mechanisms of the effects of MSCs on pulmonary vascular function remain unknown. It is believed that nitric oxide (NO) and endothelial nitric oxide synthase (eNOS) play an essential role in the regulation of vascular function and homeostasis. In the present study, we investigated the effect of adipose tissue-derived stem cells (ADSCs) on pulmonary microvascular endothelial cells (PMVECs) and the lung in a lipopolysaccharide (LPS)-induced ALI model in vitro and in vivo. Our results showed that ADSCs were able to attenuate the severity of ALI and pulmonary edema. Increased expression of the eNOS protein was also observed in pulmonary PMVECs and in the lung following treatment with ADSCs. Furthermore, ADSCs increased the concentration of eNOSderived NO to remodel ALI. The results suggest that ADSCs may be a promising candidate for ALI treatment through interaction with eNOS and eNOS-derived NO.
\end{abstract}

\section{Introduction}

Acute lung injury (ALI) is a common complication following sepsis. Despite advances in supportive care and pharmacologic treatment, mortality from ALI remains unacceptably high (1). Previous studies have demonstrated that mesenchymal stem cells (MSCs) have therapeutic effects on ALI. MSCs are able to attenuate pulmonary edema and inflammatory factors, but enhance anti-inflammatory cytokine (2). MSCs can be engrafted in injured lung tissue to differentiate into epithelial cells types, which are closed to alveolar fluid transport (3-5).

Correspondence to: Dr Qingping Wen, Department of Anesthesiology, The First Affiliated Hospital of Dalian Medical University, 222 Zhongshan Road, Dalian, Liaoning 116011, P.R. China E-mail: dmuwqp@163.com

Key words: acute lung injury, adipose tissue-derived stem cell, endothelial nitric oxide synthase, nitric oxide
However, the mechanisms of the effects of MSCs on pulmonary vascular function have yet to be fully elucidated.

MSCs can be obtained through different methods as compared with other tissues, the standard adipose tissue can easily be sampled as the source of multipotent cells, known as adipose-derived stem cells (ADSCs). A larger number of MSCs in adipose tissue can easily be harvested and rapidly proliferated. Increasing evidence suggests that adipose tissue may be a suitable source of MSCs (6).

Nitric oxide (NO) has been reported to be an essential signaling molecule with several roles that affect a number of physiological and pathophysiological functions, including angiogenesis, vascular tone, and it may also lead to endothelial tube formation (7). NO is synthesized by a family of enzymes known as NO synthases. Among these enzymes is endothelial nitric oxide synthase (eNOS). eNOS is essential to endothelial cells in keeping their functions and homeostasis. eNOS is able to modulate pulmonary arterial hypertension (8). Moreover, the reduced eNOS expression causes endothelial cells to be vulnerable to apoptosis (9). Therefore, eNOS and eNOSderived NO are considered to play a role in the regulation of vascular function.

We hypothesized that the roles of eNOS and eNOS-derived NO were important targets as contributors to pulmonary microvascular endothelial cells (PMVECs) and lung protective effects of ADSCs during ALI.

\section{Materials and methods}

Experimental animals. In the present study, adult SpragueDawley (SD) rats weighing $220 \mathrm{~g}$ were obtained from the Animal Center of Dalian Medical University, China.

Isolation and culture of ADSCs. ADSCs were isolated from human adipose tissue as previously reported (6). Human adipose tissue of 15-20 g was isolated from a grossly normalappearing region farthest away from the cancer following surgery for localized breast cancer. Adipose tissue was washed extensively with sterile phosphate-buffered saline (PBS) to remove contaminating debris, extracted blood vessels, and was cut into small fragments of tissue $\left(1 \mathrm{~mm}^{3}\right)$. The small fragments of tissue were treated with $1 \%$ collagenase I in PBS for $1 \mathrm{~h}$ at $37^{\circ} \mathrm{C}$ with gas bath and gentle agitation. The cellular pellet was resuspended in high glucose DMEM (Gibco, USA) supple- 
mented with 15\% FBS (Gibco) and 1\% penicillin-streptomycin. ADSCs were used for all experiments at passages 6-12.

Isolation and culture of PMVECs. Rat PMVECs were isolated from rat lung as previously reported (10). PMVECs were isolated from an $\sim 80 \mathrm{~g}$ SD rat which was anesthetized and sacrificed by bloodletting. Rat lungs were perfused with D-Hank's solution to whiten them from the right ventricles. Peripheral lung tissue was isolated and cut into small fragments of tissue $\left(1 \mathrm{~mm}^{3}\right)$. The small fragments of tissue were seeded in a $25-\mathrm{cm}^{2}$ flask. Subsequently, the tissue fragments were incubated in low glucose DMEM (Gibco) supplemented with $20 \%$ FBS and $1 \%$ penicillin-streptomycin. PMVECs were used for experiments at passages 5-8. The PMVECs were identified by von Willebrand factor (vWF) and KDR (11).

Experimental design. In vitro, PMVECs were treated with $5 \mu \mathrm{g} / \mathrm{ml}$ lipopolysaccharide (LPS) (Sigma, USA) for $4 \mathrm{~h}$ at $37^{\circ} \mathrm{C}$, and then washed with sterile PBS. In the co-culture group, PMVECs were co-cultured with ADSCs in either a standard single well or in a Transwell $(0.4 \mu \mathrm{m}$ pore size; Costar) for $72 \mathrm{~h}$. In the control group, PMVECs were cultured without ADSCs. In vivo, rats were randomly assigned to three groups: the sham group $(n=5)$, the ALI group $(n=10)$ and the ADSC group $(n=10)$. Sham group rats received normal saline instead of LPS or ADSCs in the same manner and served as control. The ALI group rats received an intraperitoneal injection of $6 \mathrm{mg} / \mathrm{kg}$ LPS to induce ALI. The ADSC group rats were injected with $\sim 5 \times 10^{5}$ ADSCs through the caudal vein. ALI group rats received normal saline instead of ADSCs. All animals were used to test at 7 days.

Flow cytometry. Flow cytometry for expression of a panel of surface markers was performed using a BD Biosciences FACS machine using standard techniques. ADSCs were harvested and washed with PBS, and stained by PE-CD34, FITC-CD90 and PE-106 antibodies (BD Biosciences, USA).

ADSC differentiation into vascular endothelial cells. Human ADSCs were plated on 6-well plates at a density of $\sim 1.2 \times 10^{4}$ cells/plate. Before plating, the 6 -well plates were coated with human fibronectin (Millipore, USA). Human ADSCs were supplemented with low glucose DMEM, $5 \% \mathrm{FBS}, 40 \mathrm{ng} / \mathrm{ml}$ vascular endothelial growth factor (VEGF) (PeproTech, Inc., USA) and 1\% penicillin-streptomycin for 7 days.

Immunofluorescence staining. The cells were fixed in $4 \%$ paraformaldehyde at $4^{\circ} \mathrm{C}$ overnight, and permeabilized with $0.1 \%$ Triton X-100 prior to the addition of antibodies for $15 \mathrm{~min}$. Primary antibodies included vWF (1:50 dilution; Wuhan Sanying Biotechnology, Inc., China), KDR (1:50 dilution), eNOS (1:50 dilution) (both from Santa Cruz Biotechnology, Inc., Santa Cruz, CA, USA) and were incubated for $2 \mathrm{~h}$. FITC-labeled secondary antibodies were incubated for $1.5 \mathrm{~h}$. Hoechst 33258 was used to trace the nucleus and were incubated for $15 \mathrm{~min}$. The immune complex was carried out using fluorescent microscopy.

NO measurement. Culture medium was collected at 24,48 and $72 \mathrm{~h}$; the plasma of rats was collected at 7 days. All samples were analyzed for $\mathrm{NO}$ production using an $\mathrm{NO}$ assay kit. These analyses were carried out in accordance with the manufacturer's instructions (Nanjing Jiancheng Bioengineering Institute, China).

Lung wet-to-dry ratio. A lung wet-to-dry weight ratio (W/D ratio) was valued as the degree of pulmonary edema. Lung wet weight was determined immediately after removal of the left lung. Lung dry weight was determined after the lung had been dried at $60^{\circ} \mathrm{C}$ for $48 \mathrm{~h}$, and the W/D ratio was calculated by division.

Western blot analysis. Total proteins were extracted from the rat inferior lobe of the right lung. The proteins were loaded onto a linear gradient polyacrylamide gel of $10 \%$ with a stacking gel of $4 \%$ polyacrylamide, and subjected to electrically transfer onto a PVDF membrane following electrophoresis. Membranes were blocked by incubation with blocking buffer containing 5\% skimmed powdered milk for $1 \mathrm{~h}$, followed by incubation with primary antibodies at $4^{\circ} \mathrm{C}$ overnight. Primary antibodies including eNOS (1:250 dilution), inducible nitric oxide synthase (iNOS) (1:200 dilution), $\beta$-actin (1:400 dilution) (all from Santa Cruz Biotechnology, Inc.) were used. After washing, the membranes were incubated with anti-IgG horseradish peroxidase-linked secondary antibodies (1:6,000 dilution). After detecting a standard ECL, they were subjected to image analysis using ImageJ software program.

Statistical analysis. Results are presented as the means \pm SEM and analyzed using SPSS 17.0 software. Experimental data were analyzed using one-way analysis of variance (ANOVA) except for the quantitative analysis of eNOS expression in PMVECs which was performed using Student's t-test. $\mathrm{P}<0.05$ was considered to indicate statistically significant differences.

\section{Results}

Characteristics of ADSCs in culture. The majority of cells displayed a spindle-like shape or fibroblast-like shape (Fig. 1A-C). Flow cytometry analysis demonstrated expression of ADSC surface markers. ADSCs were positive for expression of CD90 that expressed $\sim 90.6 \%$, but showed low expression for CD106 and CD34, which expressed 7.5 and $1.8 \%$, respectively (Fig. 1D-F).

Characteristics of PMVECs in culture. The majority of cells displayed a cobblestone-like shape (Fig. 2A). Immunofluorescence analysis demonstrated that $>95 \%$ PMVECs were positive for expression of vWF and KDR (Fig. 2B and C).

ADSCs differentiate into vascular endothelial cells. Under the inductive conditions, the ADSCs showed morphology of vascular endothelial cells and expressed vWF and KDR (Fig. 3A-C), which are vascular endothelial lineage-specific markers. By contrast, ADSCs under control conditions (noninduction) did not express these markers.

ADSCs enhance eNOS expression in PMVECs following co-culture. PMVECs were co-cultured with ADSCs in Transwell for $72 \mathrm{~h}$. In the co-culture group, eNOS expression 

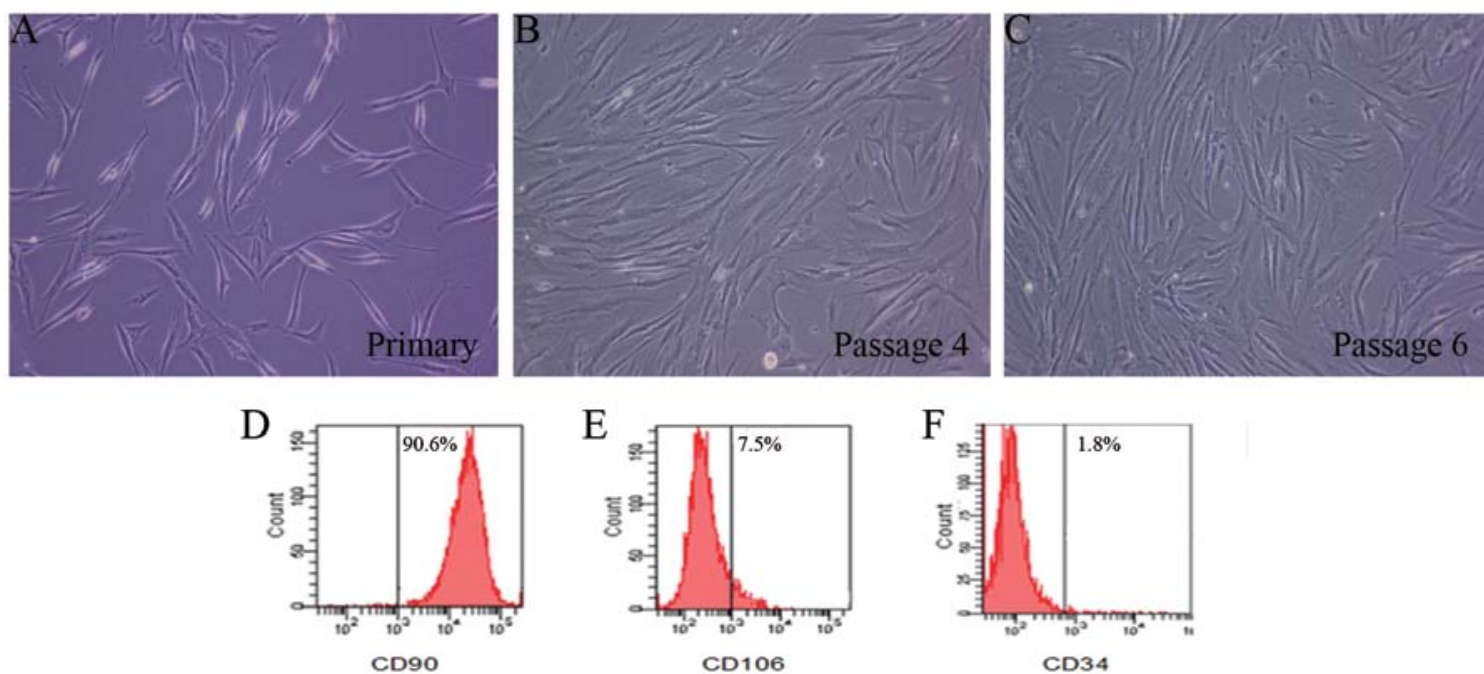

Figure 1. Characteristics of ADSCs. (A-C) Phase-contrast micrographs of ADSCs were observed. (D-F) Flow cytometry analysis demonstrated the expression of ADSC surface markers.
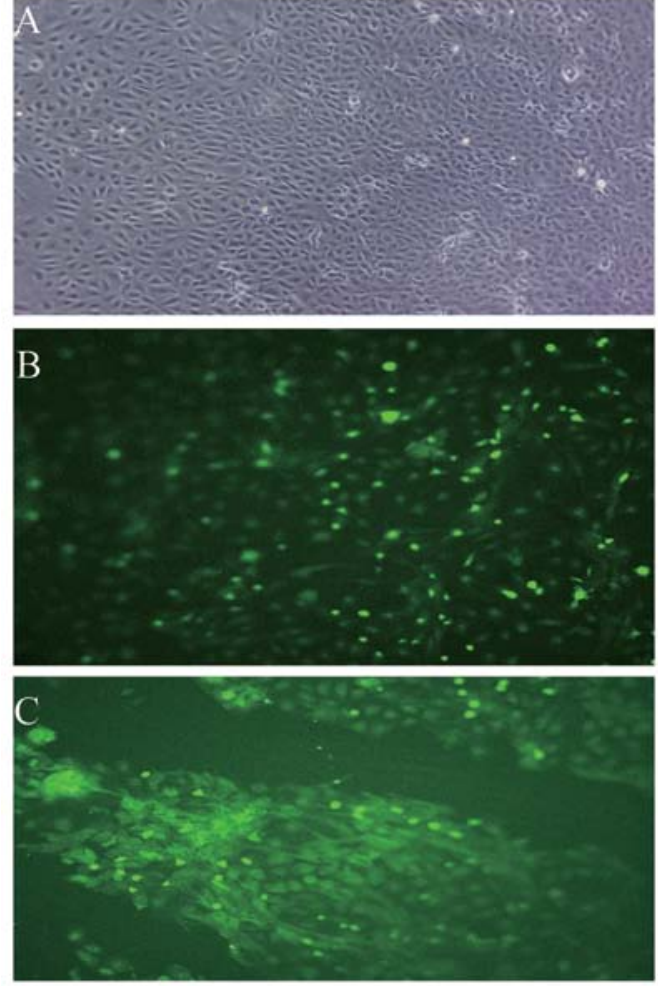

Figure 2. Characteristics of pulmonary microvascular endothelial cells PMVECs were isolated from rat lung tissue. (A) Phase-contrast micrographs of primary PMVECs were observed at 4 days. (B and C) Immunofluorescence showed that PMVECs were positive for expression of vWF and KDR.

increased significantly compared to the control group at $72 \mathrm{~h}$ (Fig. 4A and B). Meanwhile, ADSCs showed morphology of vascular endothelial lineage cells and expressed vWF following co-culture with PMVECs (Fig. 4C). ADSCs differentiated into vascular endothelial lineage cells.

NO concentration is higher in the co-culture medium. The concentrations of NO were measured at 24,48 and $72 \mathrm{~h}$. There was no difference between the control group and the
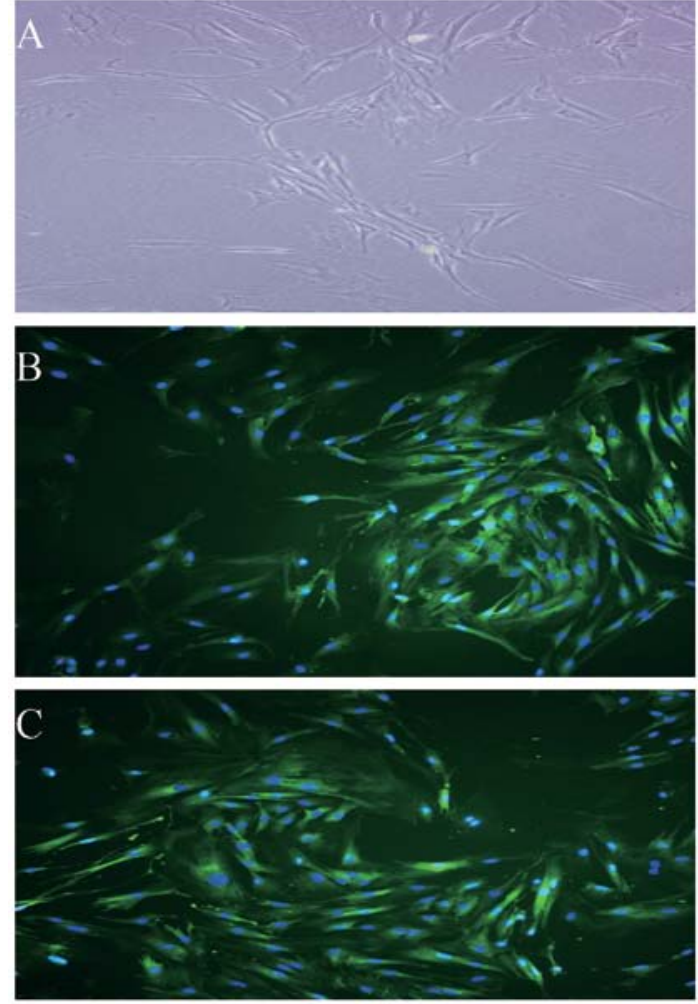

Figure 3. (A) ADSCs differentiate into vascular endothelial cells. (B and C) ADSCs showed morphology of vascular endothelial cells and expressed vWF and KDR.

co-culture group at the initial 24 and $48 \mathrm{~h}$. The concentration of $\mathrm{NO}$ was higher in the co-culture group than in the control group at $72 \mathrm{~h}(\mathrm{P}<0.05)$, and the co-culture group was similar to their baseline (Fig. 5).

ADSCs reduce lung W/D ratio. Results showed that the lung W/D ratio of rats treated with ADSCs decreased significantly compared with the ALI group at 7 days $(\mathrm{P}<0.05)$. The lung W/D ratio of the ADSC group was similar to the sham group 

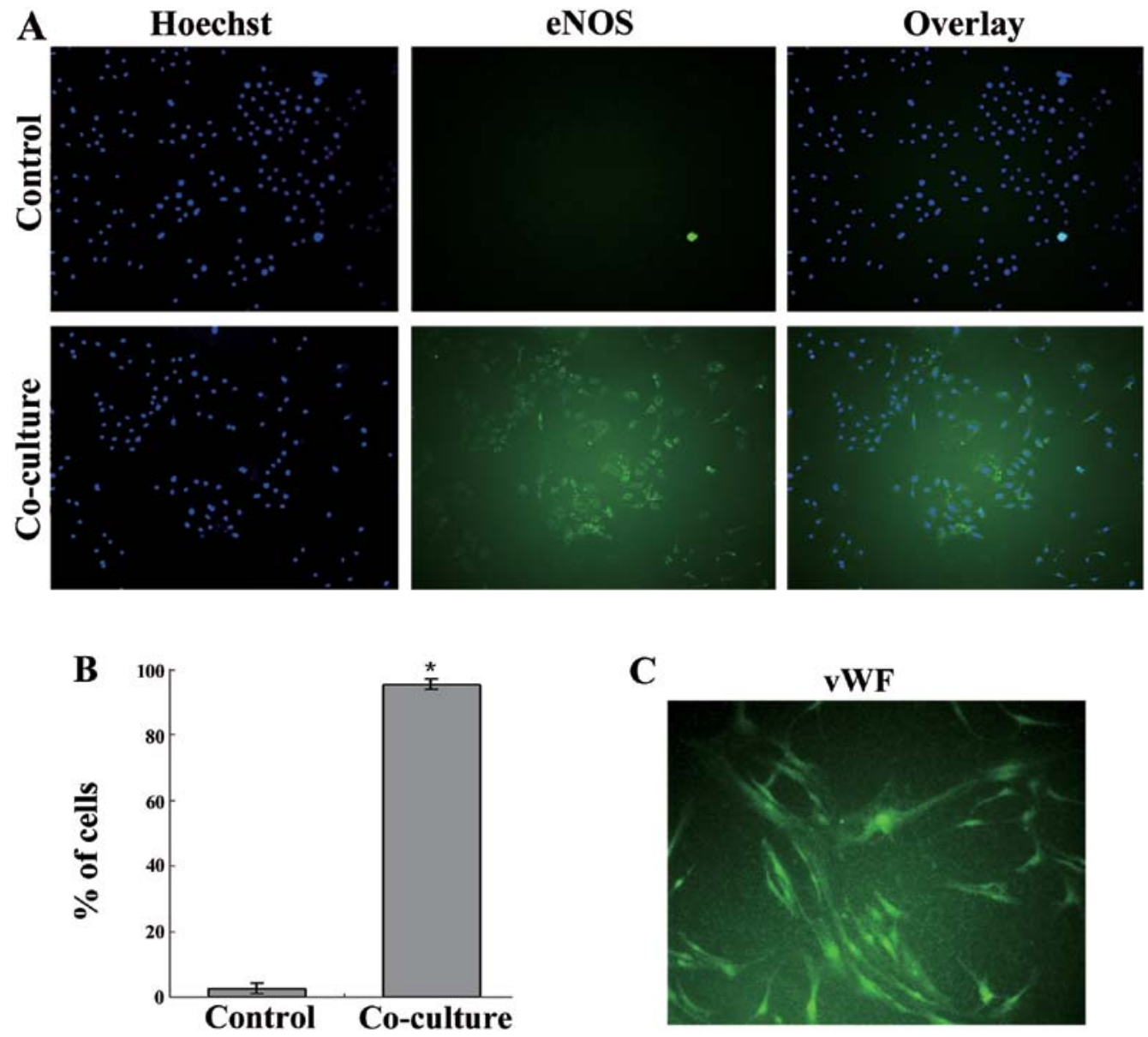

Figure 4. ADSCs enhance eNOS expression in PMVECs. (A) In the co-culture group, eNOS expression in PMVECs increased significantly as compared to the control group at $72 \mathrm{~h}$. (B) Quantitative analysis. Four independent experiments were performed in each group. (C) "P<0.05. The ADSCs differentiated into vascular endothelial lineage cells.

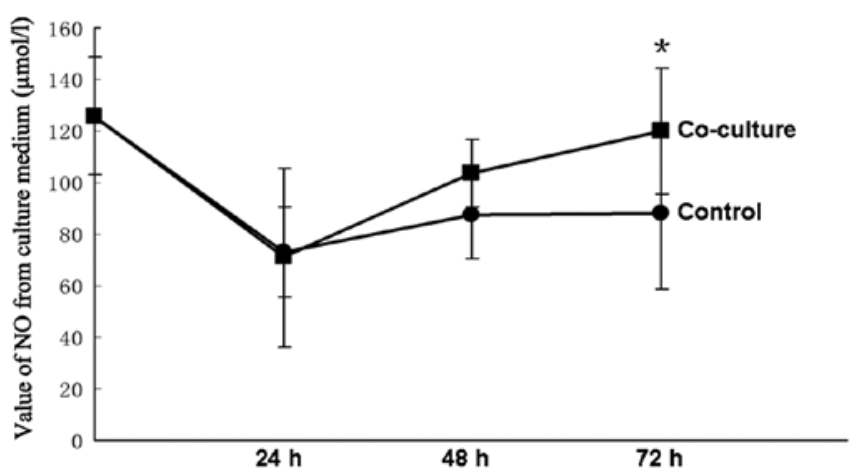

Figure 5. NO is highly produced in the co-culture medium. ${ }^{*} \mathrm{P}<0.05$, co-culture vs. control group; $\mathrm{n}=5$ in each group.

(Fig. 6). Our results indicated that ADSCs could reduce the severity of lung injury and pulmonary edema.

ADSCs enhance eNOS protein expression in the lung. By transplantation of ADSCs, eNOS protein expression increased significantly in the ADSC group compared with the ALI group at 7 days $(\mathrm{P}<0.05)$, but the ADSC group was similar to the sham group (Fig. 7). Our results indicated that ADSCs restored and enhanced eNOS, which contributed to protect and remodel ALI.

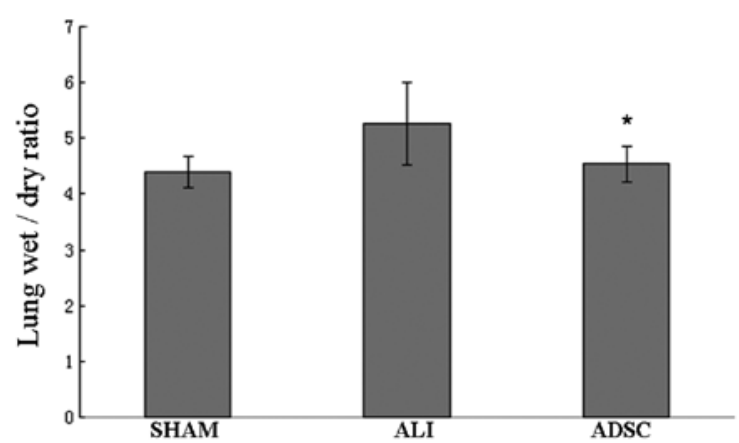

Figure 6. ADSCs reduce lung W/D ratio. ADSCs reduced the severity of lung injury and pulmonary edema. ${ }^{*} \mathrm{P}<0.05$, ADSC vs. ALI group; sham group, $\mathrm{n}=5$; ALI group, $\mathrm{n}=10$; ADSC group, $\mathrm{n}=10$.

ADSCs attenuate the concentration of $N O$, possibly due to the attenuation of iNOS protein expression in vivo. In the ADSC group, the concentration of NO decreased significantly compared with the ALI group at 7 days $(\mathrm{P}<0.05)$, but the ADSC group was similar to the sham group (Fig. 8). Moreover, the iNOS protein expression decreased significantly in the ADSC group compared with the ALI group at 7 days $(\mathrm{P}<0.05)$, but the ADSC group was similar to the sham group (Fig. 9). 


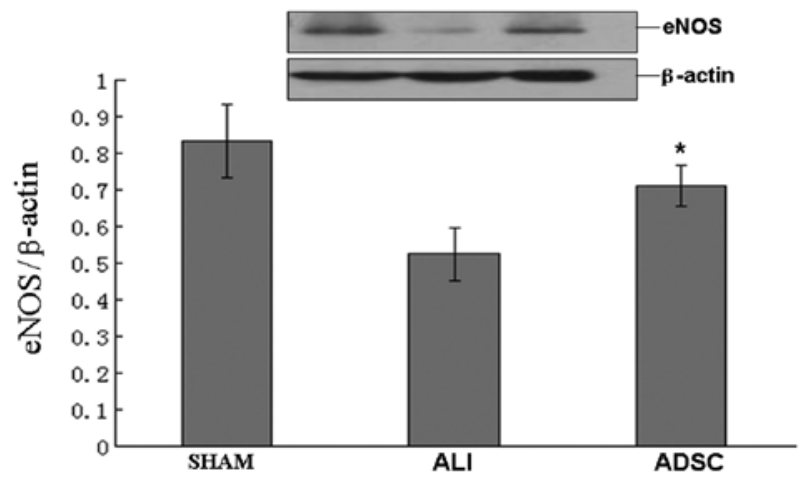

Figure 7. ADSCs restore and enhance eNOS protein expression in the lung, which contribute to protect and remodel ALI. ${ }^{*} \mathrm{P}<0.05$, ADSC vs. ALI group; $n=4$ in each group.

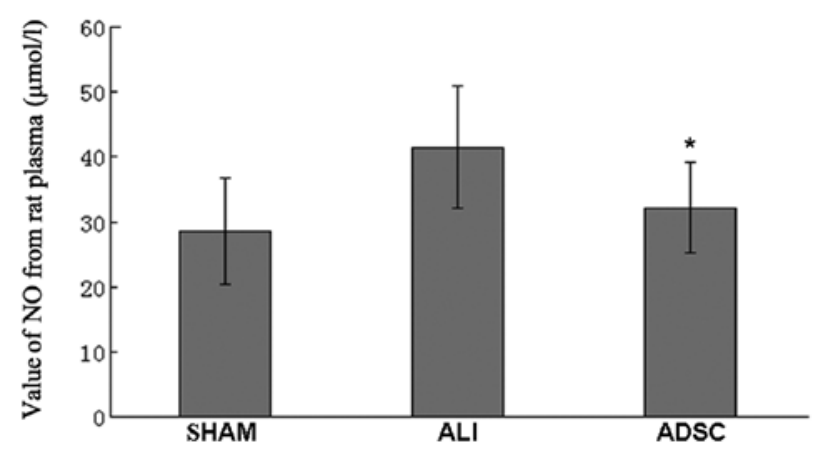

Figure 8. ADSCs attenuate the concentration of $\mathrm{NO}$ in vivo. ${ }^{*} \mathrm{P}<0.05, \mathrm{ADSC}$ vs. ALI group; sham group, $\mathrm{n}=5$; ALI group, $\mathrm{n}=10$; ADSC group, $\mathrm{n}=10$.

\section{Discussion}

The present study successfully harvested ADSCs from adipose tissue and identified their surface markers by the FACS machine. It was demonstrated that most of the ADSCs were positive to surface antigen CD90, but negative for CD34 and CD106, which confirmed previous studies (12-14). It has been reported that multi-lineage capacity of a population of stem cells are successfully isolated from human adipose tissue (6). The adipose tissues are shown to be an abundant source for isolating multipotent cells (15). In order to induce stem cell differentiation into vascular endothelial cells in vitro, the ADSCs require angiogenic factors such as VEGF, transforming growth factor- $\beta$ (TGF- $\beta$ ) and basic growth fibroblast growth factor (bFGF) (16). However, we successfully used the VEGF to induce ADSC differentiation into endothelial cells. This result demonstrated that ADSCs efficiently differentiated into vascular endothelial cells in vitro.

Hyperpermeability response and pulmonary arterial hypertension are key among most pathophysiological characteristics of ALI. The integrity of the PMVECs is essential to prevent the influx of protein-rich fluid from the plasma. When the PMVECs are destroyed by inflammation, it may further lead to high-permeability and pulmonary edema. Therefore, the injured PMVECs are a principal event to ALI. Our observations showed that the ADSCs enhanced eNOS expression in PMVECs and NO was highly produced in the co-culture medium. When PMVECs were exposed to LPS and

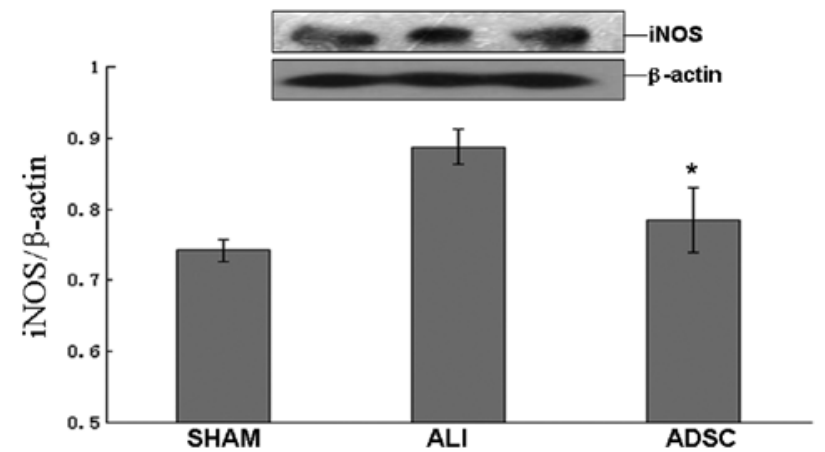

Figure 9. The decrease in the concentration of NO may be a result of the ADSC attenuation of iNOS protein expression in vivo. ${ }^{*} \mathrm{P}<0.05$, ADSC vs. ALI group; $\mathrm{n}=4$ in each group.

did not co-culture with ADSCs, eNOS expression was only slight at $72 \mathrm{~h}$. Moreover, ADSCs differentiated into vascular endothelial lineage cells in Transwell co-culture experiments. Previous studies have shown that ADSCs are capable of regenerative angiogenesis due to their differentiation into vascular endothelial cells. ADSCs secrete angiogenic factors, such as VEGF, TGF- $\beta$, bFGF, which are inductive factors that have the role in differentiating into vascular endothelial cells $(16,17)$. Furthermore, the injured PMVECs also had a limited endothelial cell capability and characteristic in Transwell co-culture experiments. ADSCs were affected by PMVECs and differentiated into vascular endothelial lineage cells to activate the secretion of eNOS-derived NO accompanied by secreted PMVECs.

Several studies have reported that MSCs are not labeled and traced in the in vivo model of ALI, but the MSCs have therapeutic effects on ALI $(2,18)$. We studied the effects of transplantation of ADSCs in rats suffering from ALI. When ADSCs were administered to ALI, ADSCs reduced the lung W/D ratio of ALI, which is a marker of the severity of lung injury and pulmonary edema. The eNOS and eNOS-derived NO signaling played an essential role in resisting injuries in vascular endothelial cells during ALI.eNOS and eNOS-derived $\mathrm{NO}$ are fundamental factors in eliciting the hyperpermeability response in experiments $(19,20)$. The NO-soluble guanylate cyclase (sGC)-cyclic guanosine monophosphate (cGMP) signaling pathway plays a critical role in the regulation of pulmonary vascular tone and resistance in pulmonary arterial hypertension. The effects of sGC stimulators and activators depend on ongoing eNOS-derived NO generation (21). LPS abolishes acetylcholine-induced relaxation mediated by endothelium-derived hyperpolarizing factor, although partially inhibited the eNOS-derived NO mediate relaxation component in the rat pulmonary artery (22). Our data showed that eNOS protein expression increased in lung tissue after administering ADSCs. Therefore, it was considered that relieving pulmonary vascular injuries depended on eNOS expression by administering ADSCs. We found that eNOS-derived NO signaling was activated, but the concentration of NO was decreased significantly in vivo. To explain this phenomenon, we consider that several cell types are involved in ALI, such as alveolar epithelial cell types I and II, vascular endothelial cells, smooth muscle cells, fibroblasts, neutrophils and macrophages. Macrophages, in particular, play a significant role in 
the pathophysiology of LPS-induced ALI. eNOS produces low amounts of $\mathrm{NO}$ and activities in endothelial cells. However, iNOS activates in macrophages and produces large amounts of NO following stimulation by LPS. It is believed that iNOS and iNOS-derived NO mediate the pathophysiological processes of ALI, including pulmonary neutrophil infiltration, oxidant stress and pulmonary arterial hypertension. PMVEC barrier dysfunction is caused by iNOS came from alveolar macrophage (23). We observed that iNOS protein and iNOSderived NO concentration decreased significantly following delivery of ADSCs. Therefore, our evidence demonstrated that decreased iNOS was a part of the protection from ALI provided by ADSCs.

We suggest that ADSCs restored and enhanced eNOS and eNOS-derived NO, which contributed to protect and remodel ALI. The ADSCs decreased iNOS, which was a part of protection during ALI. Collectively, ADSCs may be a promising therapeutic strategy for ALI.

\section{Acknowledgements}

This study was supported by the National Natural Science Fund of China (no. 81273923).

\section{References}

1. Rubenfeld GD, Caldwell E, Peabody E, et al: Incidence and outcomes of acute lung injury. N Engl J Med 353: 1685-1693, 2005.

2. Gupta N, Su X, Popov B, et al: Intrapulmonary delivery of bone marrow-derived mesenchymal stem cells improves survival and attenuates endotoxin-induced acute lung injury in mice. J Immunol 179: 1855-1863, 2007.

3. Ortiz LF, Gambelli C, McBride D, et al: Mesenchymal stem cell engraftment in lung is enhanced in response to bleomycin exposure and ameliorates its fibrotic effects. Proc Natl Acad Sci USA 100: 8407-8411, 2003.

4. Rojas M, Xu J, Woods CR, et al: Bone marrow derived mesenchymal stem cells in repair of the injured lung. Am J Respir Cell Mol Biol 33: 145-152, 2005.

5. Kotton DN, Ma BY, Cardoso WV, et al: Bone marrow-derived cells as progenitors of lung alveolar epithelium. Development 128: 5181-5188, 2001.

6. Zuk PA, Zhu M, Mizuno H, et al: Multilineage cells from human adipose tissue: implications for cell-based therapies. Tissue Eng 7: 211-228, 2001.

7. Ito $\mathrm{H}$, Matsushita S, Ishikawa $\mathrm{S}$, et al: Significant correlation between endothelial nitric oxide synthase (eNOS) expression and alveolar repair in elastase-induced rat pulmonary emphysema. Surg Today 43: 293-299, 2013.
8. Maron BA, Zhang YY, White K, et al: Aldosterone inactivates the endothelin-B receptor via a cysteinyl thiol redox switch to decrease pulmonary endothelial nitric oxide levels and modulate pulmonary arterial hypertension. Circulation 126: 963-974, 2012.

9. Hoffmann J, Haendeler J, Aicher A, et al: Aging enhances the sensitivity of endothelial cells toward apoptotic stimuli: important role of nitric oxide. Circ Res 89: 709-715, 2001.

10. Ryan US, White LA, Lopez M and Ryan JW: Use of microcarriers to isolate and culture pulmonary microvascular endothelium. Tissue Cell 14: 597-606, 1982.

11. Solodushko V, Parker JC and Fouty B: Pulmonary microvascular endothelial cells form a tighter monolayer when grown in chronic hypoxia. Am J Respir Cell Mol Biol 38: 491-497, 2008.

12. Chieregato K, Castegnaro S, Madeo D, et al: Epidermal growth factor, basic fibroblast growth factor and platelet-derived growth factor-bb can substitute for fetal bovine serum and compete with human platelet-rich plasma in the ex vivo expansion of mesenchymal stromal cells derived from adipose tissue. Cytotherapy 13: 933-943, 2011.

13. Katz AJ, Tholpady A, Tholpady SS, Shang H and Oqle RC: Cell surface and transcriptional characterization of human adiposederived adherent stromal (hADAS) cells. Stem Cells 23: 412-423, 2005.

14. De Ugarte DA, Alfonso Z, Zuk PA, et al: Differential expression of stem cell mobilization-associated molecules on multi-lineage cells from adipose tissue and bone marrow. Immunol Lett 89: 267-270, 2003

15. Lee RH, Kim B, Choi I, et al: Characterization and expression analysis of mesenchymal stem cells from human bone marrow and adipose tissue. Cell Physiol Biochem 14: 311-324, 2004.

16. Rehman J, Traktuev D, Li J, et al: Secretion of angiogenic and antiapoptotic factors by human adipose stromal cells. Circulation 109: 1292-1298, 2004.

17. Miranville A, Heeschen C, Sengenes C, et al: Improvement of postnatal neovascularization by human adipose tissue-derived stem cells. Circulation 110: 349-355, 2004.

18. Burnham EL, Taylor WR, Quyyumi AA, et al: Increased circulating endothelial progenitor cells are associated with survival in acute lung injury. Am J Respir Crit Care Med 172: 854-860, 2005.

19. Hatakeyama T, Pappas PJ, Hobson RW, et al: Endothelial nitric oxide synthase regulates microvascular hyperpermeability in vivo. J Physiol 574: 275-281, 2006.

20. Sanchez FA, Savalia NB, Duran RG, et al: Functional significance of differential eNOS translocation. Am J Physiol Heart Circ Physiol 291: H1058-H1064, 2006.

21. Lang M, Kojonazarov B, Tian X, et al: The soluble guanylate cyclase stimulator riociguat ameliorates pulmonary hypertension induced by hypoxia and SU5416 in rats. PLoS One 7: e43433, 2012.

22. Subramani J, Leo MD, Kathirvel K, et al: Essential role of nitric oxide in sepsis-induced impairment of endothelium-derived hyperpolarizing factor mediated relaxation in rat pulmonary artery. Eur J Pharmacol 630: 84-91, 2010

23. Farley KS, Wang LF, Law $C$ and Mehta S: Alveolar macrophage inducible nitric oxide synthase-dependent pulmonary microvascular endothelial cell septic barrier dysfunction. Microvasc Res 76: 208-216, 2008. 